\title{
Schnittstelle psychiatrische Akutstationen und betreute Wohneinrichtungen - Charakteristika von Patienten mit langen Klinikaufenthalten
}

\author{
Jäger, Matthias ; Theodoridou, Anastasia ; Hoff, Paul ; Rössler, Wulf
}

DOI: https://doi.org/10.1055/s-0033-1349461

Posted at the Zurich Open Repository and Archive, University of Zurich ZORA URL: https://doi.org/10.5167/uzh-84581

Journal Article

Accepted Version

Originally published at:

Jäger, Matthias; Theodoridou, Anastasia; Hoff, Paul; Rössler, Wulf (2013). Schnittstelle psychiatrische Akutstationen und betreute Wohneinrichtungen - Charakteristika von Patienten mit langen Klinikaufenthalten. Psychiatrische Praxis, 40:439-446.

DOI: https://doi.org/10.1055/s-0033-1349461 
Schnittstelle psychiatrische Akutstationen und betreute Wohneinrichtungen - Charakteristika von Patienten mit langen Klinikaufenthalten

Intersection of acute psychiatric wards and sheltered housing - parameters of patients with long inpatient episodes

Matthias Jäger

Anastasia Theodoridou

Paul Hoff

Wulf Rössler

Psychiatrische Universitätsklinik Zürich

Korrespondenzadresse:

Dr. med. Matthias Jäger

Lenggstrasse 31

Postfach 1931

8032 Zürich

Telefon $\quad+41(0) 443842685$

Fax $\quad+41(0) 443842506$

E-mail: matthias.jaeger@puk.zh.ch 


\section{Zusammenfassung}

Anliegen: Ermittlung soziodemographischer, klinischer und behandlungsbezogener Parameter sowie die Häufigkeit von Platzierungsaufträgen in betreute Wohnformen bei 18-65-jährigen Patienten mit langem Aufenthalt auf einer psychiatrischen Akutstation.

Methode: Fünfjährige retrospektive Kohortenstudie ( $\mathrm{N}=3.928)$.

Ergebnisse: Charakteristika von Patienten mit langem Aufenthalt weisen Gemeinsamkeiten mit denen der „Heavy user“ auf. Platzierungsauftrag bei einem Drittel dieser Patienten.

Schlussfolgerungen: Neben klinischen Faktoren ist die Übertrittsmöglichkeit in eine geeignete Wohnform wahrscheinlich ein relevanter Faktor für die Aufenthaltsdauer.

Schlüsselwörter: Behandlungsdauer, Inanspruchnahme, betreutes Wohnen, psychiatrische Versorgung 


\section{Abstract}

Objective: This paper describes socio-demographic, clinical, and treatment-related parameters of psychiatric patients who were hospitalized for at least two months on an acute psychiatric ward compared to patients with a shorter inpatient treatment episode. Furthermore, it is evaluated how frequent these long-staying patients are awaiting a room in a sheltered housing facility.

Methods: We investigated the longest inpatient treatment period of all patients aged between 18 and 65 years on an acute ward of the Psychiatric University Hospital Zurich $(\mathrm{N}=3,928)$ using the basic documentation of the years 2006 to 2010 .

Results: $20 \%$ of all patients on acute wards had a stay of more than 60 days. Socio-demographic and clinical characteristics are similar to those of "heavy users" of mental health services. Social work is involved more frequently, and placement in sheltered housing facilities is intended in one third of those patients.

Conclusions: A substantial part of the patients who stay at least once longer than two months on an acute ward are discharged to sheltered housing. Besides severity of illness it is likely that lack of availability of an adequate housing option contributes to length of stay. Intensified cooperation of the psychiatric clinic with sheltered housing facilities as well as alternative options for those in need of assisted housing and mental health care might help to reduce their extensive usage of inpatient treatment capacities. Interventions and services have to be adapted to local conditions.

Key words: length of stay, sheltered housing, assisted accommodation, mental health services 


\section{Konsequenzen für Klinik und Praxis}

- Ungeachtet der Abnahme der durchschnittlichen fallbezogenen Aufenthaltsdauer auf psychiatrischen Akutstationen benötigt ein substanzieller Anteil der Patienten unverändert ein hohes Maß an stationären Behandlungskapazitäten.

- Hierfür ist neben dem Schweregrad der Erkrankung vielfach eine soziale Belastung, insbesondere im Bereich Wohnen ausschlaggebend.

- Dies stellt eine Herausforderung für die Versorgung an der Schnittstelle zwischen Kliniken und nachsorgenden Hilfsangeboten dar und erfordert alternative multiprofessionelle Angebote zur Verbesserung der Versorgungskontinuität und -qualität und zu einer Reduktion der Inanspruchnahme stationärer akutpsychiatrischer Behandlung. 


\section{Einleitung}

Die stationäre Behandlungsdauer psychiatrischer Patienten ist durch die hohe Korrelation mit den Fallkosten einer der meist beachteten Indikatoren seitens der Kliniken und insbesondere der Kostenträger [1]. Als Qualitätsmerkmal für die seit den 1970er Jahren angestrebte Deinstitutionalisierung in der psychiatrischen Versorgung kommt der Verweildauer zusätzliche Bedeutung zu. Ungeachtet der Kontroverse um die Behandlungsdauer als Effizienzkriterium beziehungsweise Qualitätsindikator des Versorgungssystems nimmt diese Jahr um Jahr bei stabiler Patientenzahl ab, während die Wiederaufnahmeraten konsekutiv steigen [2-4].

Gründe für sinkende Aufenthaltsdauern sind in der zunehmenden Leistungsverdichtung, dem Ausbau teilstationärer, ambulanter und komplementärer Versorgungsstrukturen und dem Druck zur Reduktion von Fallkosten zu finden. Eine verlässliche Prädiktion der Aufenthaltsdauer im Einzelfall durch beispielsweise krankheitsbezogene Merkmale, insbesondere die psychopathologische Symptomatik oder ICD-10-Diagnose, gelang nicht [5, 6]. Es sind allerdings einige Faktoren bekannt, die mit höheren Aufenthaltsdauern und Wiederaufnahmeraten assoziiert sind. Neben soziodemographischen und klinischen Variablen, die nur einen kleinen Beitrag zur Varianzaufklärung leisten, sind vor allem Aspekte des Behandlungsverlaufs (zum Beispiel Zwangsmaßnahmen) und institutionelle Merkmale zu nennen $[7,8]$.

Diejenigen Patienten, die den größten Anteil stationärer Behandlungskapazitäten in Anspruch nehmen (je nach Definition ist das Kriterium die Anzahl der Aufenthalte oder die kumulative Aufenthaltsdauer) werden in der Literatur auch als „Heavy user" bezeichnet. Es handelt sich dabei um rund 10\% der stationären Patienten, die bis zu $50 \%$ der Behandlungstage beziehungsweise der stationären Behandlungskosten beanspruchen [9-11]. Die Merkmale dieser Patienten sind inhomogen, jedoch spielen neben schwerer psychiatrischer Symptomatik [12, 13] auch ein hohes Ausmaß sozialer Belastungsfaktoren und mangelnde Versorgungsalternativen zu stationären Angeboten eine große Rolle für die hohe Inanspruchnahme [10, 14, 15]. Insbesondere eine adäquate Wohnform zu finden und zu halten, stellt für Patienten mit schwerer psychiatrischer Symptomatik und hoher Inanspruchnahme stationärer Behandlung eine große Herausforderung dar [16]. Obwohl das Angebot an begleiteten, betreuten und therapeutischen Wohnformen in den letzten Jahrzehnten gewachsen ist, kann eine Platzierung dieser Patientengruppe mit erheblichen Problemen verbunden sein. Über den Zusammenhang einer langen akutpsychiatrischen Behandlungsdauer mit Problemen bei der Platzierung in geeignete Wohneinrichtungen ist kaum etwas bekannt. Die vorliegende Arbeit 
untersucht daher die Charakteristika von Patienten, die mindestens einen langen Aufenthalt (> 2 Monate) auf psychiatrischen Akutstationen benötigten. Es wird angenommen, dass diese Patienten wesentliche Merkmale der „Heavy user“ erfüllen und vielfach ein Platzierungsauftrag besteht.

\section{Methoden}

Die Untersuchung basiert auf einer retrospektiven Auswertung der Basisdokumentationsdaten (PSYREC) aller Patienten zwischen 18 und 65 Jahren, die in den Jahren 2006 bis 2010 in der Psychiatrischen Universitätsklinik Zürich (PUK) stationär behandelt wurden (18.511 Fälle bei 9.900 behandelten Patienten). Zunächst wurde für jeden Patienten nur der jeweils längste Behandlungsfall innerhalb des Untersuchungszeitraums ausgewählt, sodass kein Patient mehrfach in die Stichprobe aufgenommen wurde. Eingeschlossen wurden dann diejenigen Patienten, deren längster Fall auf einer Akutstation beendet wurde $(\mathrm{N}=3.928)$. Die Auswahl erfolgte ausschließlich nach dem Kriterium der Station auf der die Behandlung stattfand und nicht nach patientenbezogenen Kriterien. Mit „Akutstation“ sind hier Aufnahmestationen gemeint, die geschlossen geführt werden können und über mindestens ein Isolierzimmer verfügen. Für die Aufnahme auf diese Stationen bestehen keine Ausschlusskriterien. Die Begründung für die Auswahl der Akutstationen liegt in der Feststellung, dass es eine Gruppe von Patienten auf diesen Stationen gibt, die auch nach einem längeren (über eine Akutbehandlung hinaus gehenden) Zeitraum weder entlassen noch auf eine spezialisierte Station oder in eine rehabilitativ orientierte Klinik verlegt werden können. Im stationären Versorgungsbereich für Erwachsene der PUK Zürich gab es im Zeitraum der Datenerhebung 8 solche Stationen bei insgesamt 15 Bettenstationen. Die anderen sieben Stationen bieten spezialisierte Behandlungen für Angst und Depressionen, Suchtpatienten sowie Frauen mit Persönlichkeitsstörungen und schwerer Traumatisierung. Alle diese Stationen werden offen geführt, sodass akute Selbst- und Fremdgefährdung als Ausschlusskriterien gelten. Die Versorgung im Kanton Zürich ist sektoriell organisiert. Die PUK ist die größte Versorgungsklinik mit einem Einzugsgebiet von rund 485.000 Einwohnern. Es findet keine Selektion der Klientel statt.

Die Untersuchung erfolgte entsprechend den Anforderungen der Deklaration von Helsinki. Die Datenselektion und die Auswertung erfolgten anonymisiert. 
Die Variable „Aufenthaltsdauer“ wurde in 3 Kategorien unterteilt (Aufenthaltsdauer bis 30 Tage, 31-60 Tage, mehr als 60 Tage). Diese Klassierung erfolgte aus inhaltlichen Gesichtspunkten, nämlich dass auf den Akutstationen mehr als die Hälfte der stationären Behandlungsfälle weniger als einen Monat, ein erheblicher Anteil jedoch zwei und mehr Monate dauert. Daher wurde entschieden, einen Gruppenvergleich durchzuführen anstatt mit nicht-parametrischen Methoden die Assoziationen der schief verteilten Variablen Aufenthaltsdauer mit andern Variablen zu untersuchen. Die für die Fragestellung relevanten Variabeln wurden aus der PSYREC-Dokumentation ausgewählt. Folgende Variablen wurde berücksichtigt: soziodemographische Variablen (Alter, Geschlecht, Nationalität, Zivilstand, Aufenthaltsort vor Aufnahme und nach Entlassung, Erwerbstätigkeit vor Aufnahme und nach Entlassung, abgeschlossener Ausbildungsgang, Finanzierung des Lebensunterhalts vor Aufnahme), klinische Variablen (Schweregrad der Erkrankung bei Aufnahme, Zustand bei Entlassung im Vergleich zur Aufnahme, Funktionsniveau bei Aufnahme und Entlassung, Hauptdiagnose bei Entlassung, Anzahl Hospitalisationen), behandlungsbezogene Variablen (Aufnahmemodus freiwillig oder per fürsorgerischer Freiheitsentziehung (analog Einweisung nach PsychKG), Initiative zur Einweisung, Verlegung während des Aufenthaltes, Zwangsmaßnahme, wichtigste Behandlung, Entscheid für Entlassung, Versicherungsklasse). Bei der Mehrheit der Variablen handelt es sich um kategoriale Größen mit nominalem Messniveau. Nach der deskriptiven Auswertung wurden teilweise mehrere Kategorien einer Variablen nach inhaltlichen Gesichtspunkten zusammengefasst, um eine größere Übersichtlichkeit zu erreichen. Die Variablen „Alter“, „Schweregrad der Erkrankung bei Aufnahme“ (Clinical Global Impression Severity Scale, CGI-S) [17], „Zustand bei Entlassung im Vergleich zu Aufnahme“ (Clinical Global Impression Improvement Scale, CGI-I), „Funktionsniveau bei Aufnahme und Entlassung“ (Global Assessment of Functioning, GAF) [18] erfüllen die Kriterien intervallskalierter normalverteilter Variablen (Schiefe/Kurtosis, Q-Q-Diagramm). Die Variable „Anzahl Hospitalisationen“ wurde in eine nominale Variable mit 3 Kategorien transformiert.

Die statistische Auswertung erfolgte mit SPSS Statistics, Version 19 (SPPS Inc., Chicago, USA). Die Vergleiche zwischen den nach der Aufenthaltsdauer klassierten Subgruppen wurden mit Varianzanalyse (ANOVA) bei stetigen Variablen und Kreuztabellen mit Chi-Quadrat-Test bei kategorialen Variablen durchgeführt. Die Bewertung der Relevanz für signifikante Gruppenunterschiede orientierte sich an den standardisierten Residuen (Werte $>$ |2I). Angesichts des 
Problems der Alpha-Fehler-Kumulierung durch multiples Testen wurden nur Ergebnisse mit einem pWert $<0,001$ (Bonferroni-Korrektur) als signifikant angenommen.

Um auf die Frage zu fokussieren, inwieweit sich diejenigen Patienten mit sehr langer Aufenthaltsdauer von denen unterscheiden, welche die durchschnittliche Aufenthaltsdauer nicht wesentlich übersteigen, wurde für die folgenden Auswertungen die Gruppe der Patienten mit Aufenthaltsdauer von 31 bis 60 Tagen herausgefiltert. Um die Bedeutung einzelner Variablen für eine lange gegenüber einer kurzen Verweildauer besser abschätzen zu können, wurde als Maß für die relative Effektstärke der kategorialen Variablen im Einzelmodell Cramer's $V$ berechnet. Werte ab 0,3 können hierbei als mittlere und Werte ab 0,5 als große Effektstärken betrachtet werden. Für die intervallskalierten Variablen wurde als Maß für die relative (von der Stichprobengröße unabhängige) Effektstärke der Korrelationskoeffizient r (konservative Berechnung nach Spearman) verwendet.

Unter Berücksichtigung der Resultate der deskriptiven Auswertung wurden anschließend diejenigen Variablen ausgewählt, die sich bei Patienten mit langen Aufenthaltsdauern (>60 Tage) von denjenigen mit kurzen Aufenthaltsdauern ( $<31$ Tage) signifikant unterschieden. Aus kategorialen Variablen wurden für jeweils relevante Kategorien dichotome Dummyvariablen gebildet. Diese wurden mit primär dichotomen und stetigen Variablen in ein binär logistisches Regressionsmodell eingegeben, um wesentliche Faktoren für eine lange Aufenthaltsdauer zu explorieren. Die auf Basis der bivariaten Analysen ausgewählten Variablen wurden nach inhaltlichen Gesichtspunkten in vier Blöcken eingegeben und per Vorwärtsselektion (LR) in das Modell eingeschlossen. Variablen, die nicht eingeschlossen wurden oder am Ende der Analyse die initiale Signifikanz verloren hatten, wurden im definitiven Modell wieder ausgeschlossen. Der Regressionskoeffizient $\operatorname{Exp}(B)$ wird zusammen mit dem 95\%-Konfidenzintervall als Maß der Richtung und Stärke der Assoziation mit der unabhängigen Variablen (Aufenthaltsdauer $>60$ Tage) angegeben. Dieses Maß entspricht der Odds Ratio. Nagelkerkes $\mathrm{R}^{2}$ gibt den Anteil der erklärten Varianz durch die bisherigen eingeschlossenen Variablen an, die Differenz zum vorherigen Wert beschreibt die zusätzlich erklärte Varianz durch die neu eingeschlossene Variable.

\section{Ergebnisse}

Die Aufenthaltsdauer ist eine stetige Variable mit schiefer Verteilung bei einem hohen Anteil kürzerer Aufenthalte (Range: 1-883 Tage). Die mediane Aufenthaltsdauer der untersuchten Behandlungsfälle auf den Akutstationen der PUK lag bei 18 Tagen und änderte sich zwischen 2006 und 2010 nicht. Der 
Anteil der Patienten, die in den Jahren 2006 bis 2010 mindestens einen Aufenthalt von mehr als 60 Tagen hatten, betrug knapp $17 \%(\mathrm{~N}=656)$.

Die mediane Aufenthaltsdauer in der Gesamtklinik (inklusive Spezialstationen, Alterspsychiatrie, offene Stationen) lag 2006 bei 30 Tagen und ging bis zum Jahr 2010 leichtgradig auf 28 Tage zurück. Die kumulative Aufenthaltsdauer über den fünfjährigen Untersuchungszeitraum wies einen Median von 23 Tagen auf den Akutstationen beziehungsweise 35 Tagen in der Gesamtklinik auf. Außerdem kann eine hohe Korrelation (Spearman-Rho: $r=0,39 ; p<0,0001$ ) der einfachen Aufenthaltsdauer mit der Behandlungsfallzahl (durchschnittlich 2,1 Aufenthalte; 1,3 bei Aufenthaltsdauer $<31$ Tage; 4,2 bei Aufenthaltsdauer $>60$ Tage) in diesen fünf Jahren nachgewiesen werden.

Patienten, die mehr als einen Monat in stationärer Behandlung waren, hatten mit 42 Jahren ein höheres Durchschnittsalter als Patienten mit Aufenthaltsdauern unter 30 Tagen (durchschnittlich 40 Jahre; Mittelwert 40,3, Standardabweichung 11,9, $p<0,001)$. Patienten mit einer Aufenthaltsdauer zwischen 31 und 60 Tagen unterschieden sich bezüglich des Alters nicht von Patienten mit einer Aufenthaltsdauer über 60 Tagen.

Tabelle 1 zeigt die soziodemographischen, klinischen und behandlungsbezogenen Variablen nach kategorialer Aufenthaltsdauer. Angegeben sind bei einzelnen Variablen jeweils nur Kategorien, die einen signifikanten Gruppenunterschied aufweisen. Unter den Patienten mit langer Aufenthaltsdauer finden sich mehr Schweizer und mehr ledige Patienten. Weiterhin arbeiteten Patienten mit langer Aufenthaltsdauer eher in einer Teilzeitanstellung beziehungsweise hatten häufig keine Arbeit. Dies gilt sowohl für die Zeit vor als auch nach dem Klinikaufenthalt. Ebenso ist der Anteil der Patienten mit eigenem Verdienst niedriger und der Anteil der Invalidenrentner deutlich höher. In Bezug auf die „höchste abgeschlossene Schulbildung“ zeigte sich kein Gruppenunterschied $(p=0,9)$.

Patienten mit der ICD-10-Hauptdiagnose einer Abhängigkeitserkrankung oder einer Diagnose aus dem F4-Bereich bleiben seltener mehr als 60 Tage in der Klinik. Der Anteil an Patienten mit einer Erkrankung aus dem schizophrenen Formenkreis ist bei Patienten mit langer Aufenthaltsdauer mehr als doppelt so hoch wie bei Patienten mit kurzer Aufenthaltsdauer. Patienten mit einer langen Aufenthaltsdauer sind häufiger bereits mindestens zum fünften Mal in der Klinik.

Mehr als die Hälfte (53\%) aller Patienten wurden per Fürsorgerischer Freiheitsentziehung (FFE) in der Klinik untergebracht. Der Anteil der Patienten, die per FFE in die Klinik eingewiesen wurden, ist unter Patienten mit langer Aufenthaltsdauer geringer als unter jenen mit kurzer Aufenthaltsdauer. Ein 
höherer Anteil der Patienten, die aus Institutionen eingetreten sind, bleibt länger als 60 Tage. Zudem ist der Anteil der zuvor Wohnsitzlosen unter Patienten mit langer Aufenthaltsdauer höher. Patienten, die aus nicht-psychiatrischen Kliniken zugewiesen wurden, bleiben seltener über 60 Tage.

Patienten mit längerer Aufenthaltsdauer werden häufiger klinikintern verlegt. Sie werden im Vergleich zur Patienten mit kürzeren Aufenthalten häufiger mindestens einmal isoliert, fixiert und / oder zwangsmediziert. Patienten mit langer Aufenthaltsdauer werden zu einem höherem Anteil an den verschiedenen Therapieformen inklusive Sozialarbeit angemeldet als Patienten mit kurzer Aufenthaltsdauer. Der Anteil der Patienten, die in ein Heim entlassen werden, ist unter Patienten mit langer Aufenthaltsdauer viermal höher als unter jenen mit kurzer Aufenthaltsdauer. Der größte Anteil der Patienten wird von niedergelassenen Psychiatern beziehungsweise Psychotherapeuten weiterbehandelt. Der Anteil der Patienten mit langer Aufenthaltsdauer, die in einer ambulanten oder teilstationären Institution der PUK weiterbehandelt werden ist mehr als doppelt so hoch wie bei Patienten mit kurzer Aufenthaltsdauer. Die Versicherungsklasse ist nicht mit der Aufenthaltsdauer assoziiert.

Die größten Effektstärken (Cramer's $\vee>0,25$ ) finden sich bei der Finanzierungssituation, der Anzahl der Voraufenthalte, der Hauptdiagnosekategorie, dem Aufenthaltsort nach Entlassung sowie den verschiedenen besuchten Therapieformen inklusive Sozialarbeit.

Der Schweregrad der Erkrankung bei Aufnahme steigt mit der Aufenthaltsdauer an ( $r=0,306$; Abb. 1). Das psychosoziale Funktionsniveau bei Aufnahme sinkt mit der Aufenthaltsdauer $(r=-0,247)$. Gleiches gilt im Wesentlichen für das psychosoziale Funktionsniveau bei Entlassung $(r=-0,118)$. Patienten mit langer Aufenthaltsdauer verbessern sich im Verlauf des Aufenthaltes stärker (CGI-I) als Patienten mit kürzerer Aufenthaltsdauer ( $r=-0,162)$. Die größte Effektstärke unter den intervallskalierten Variablen ist bei dem Schweregrad der Erkrankung bei Aufnahme zu finden.

Die Ergebnisse der Regressionsanalyse sind in Tabelle 2 dargestellt. Patienten mit einer Aufenthaltsdauer von mehr als 60 Tagen sind gemäß der logistischen Regressionsanalyse gegenüber Patienten mit einer Aufenthaltsdauer bis maximal 30 Tage durch folgende soziodemographischen und klinischen Faktoren gekennzeichnet: Häufiger ledig, IV-Bezug, schwerer krank bei Aufnahme, 
Hauptdiagnose Schizophrenie, geringeres Funktionsniveau bei Aufnahme. Im Zusammenhang mit der Behandlung zeigen sich folgende Charakteristika: mehr Voraufenthalte in der Anamnese, Aufnahme seltener per FFE, häufigere Zwangsmaßnahmen und Verlegungen während des Aufenthaltes, die klinikinterne Sozialarbeit ist häufiger involviert, die Entlassung erfolgt häufiger in ein Wohnheim, die ambulante Nachbehandlung wird von der Institutsambulanz oder niedergelassenen Psychiatern übernommen.

Behandlungsbezogene und klinische Variablen tragen 29\% zur Varianzaufklärung bei (das gesamte Modell erklärt $43 \%$ der Varianz). Die größten Odds ratios werden bei behandlungs- und entlassungsbezogenen Variablen gefunden.

Die Patienten mit einem Aufenthalt über 60 Tagen $(\mathrm{N}=656)$ wurden bezüglich der Variable „Entlassung ins Wohnheim“ untersucht. Es erfolgte ein Gruppenvergleich mit den selben Variablen wie oben. Im Folgenden sind nur jene Variablen aufgeführt, für die signifikante Gruppenunterschiede gefunden wurden.

Die einfache Aufenthaltsdauer (Entlassung ins Wohnheim 96 Tage, in andere Wohnsituation 87 Tage, $\mathrm{p}<0,001$ ) und die kumulative Aufenthaltsdauer (Entlassung ins Wohnheim 169 Tage, in andere Wohnsituation 128 Tage, p<0,001) sowie die Zahl der Aufenthalte 2006 bis 2010 (Entlassung ins Wohnheim 4,3 Fälle, in andere Wohnsituation 3,4 Fälle, $p=0,005)$ sind bei Patienten, die in ein Wohnheim entlassen werden, deutlich höher als bei jenen, die in andere Situationen entlassen werden.

Weiterhin unterscheiden sich Patienten, die in ein Wohnheim entlassen werden von jenen, die in andere Situationen entlassen werden, im Bezug auf folgende Variablen (s. Tab. 3). Sie sind seltener verheiratet, lebten wesentlich häufiger bereits vor Klinikaufnahme in Wohnheimen, sind häufiger nicht erwerbstätig, haben seltener einen eigenen Verdienst, waren häufiger mindestens fünf Mal in der Klinik. Im Bezug auf die Hauptdiagnosen findet sich die deutlichste Diskrepanz nicht bei der Schizophrenie, die nur wenig häufiger bei Patienten mit Entlassung ins Wohnheim vorkommt. Diese Patienten haben hingegen öfter eine Abhängigkeitserkrankung als Hauptdiagnose und seltener eine affektive Störung. Der Entscheid zur Entlassung wird seltener einseitig vom Patienten entgegen der Empfehlung der Ärzte getroffen und die Sozialarbeit ist häufiger involviert. Alle weiteren Variablen 
unterscheiden sich nicht signifikant. Insbesondere zeigen sich keine Unterschiede im Bezug auf Geschlecht, Alter, Krankheitsgrad, Funktionsniveau sowie Therapieteilnahme.

\section{Diskussion}

Diese Untersuchung beschäftigt sich mit Charakteristika von Patienten mit einer langen Aufenthaltsdauer auf psychiatrischen Akutstationen, insbesondere jenen Patienten, die in eine betreute Wohneinrichtung entlassen werden. Die wesentlichen Ergebnisse der Studie lassen sich wie folgt zusammenfassen. Die mediane Aufenthaltsdauer des jeweils längsten Falls pro Patient hat sich im Untersuchungszeitraum nicht verändert. Dies steht im Gegensatz zur andauernden Reduktion der fallbezogenen Aufenthaltsdauer in den letzten Jahren und zeigt, dass es eine Patientengruppe gibt, die unverändert einen hohen Anteil stationärer Behandlungsressourcen in Anspruch nimmt. Knapp ein Fünftel aller Patienten auf psychiatrischen Akutstationen benötigt mindestens einen langen Aufenthalt von mehr als zwei Monaten. Diese Patienten weisen Gemeinsamkeiten mit der in der Literatur beschriebenen Gruppe der „Heavy user“ auf, wie eine Hauptdiagnose aus dem schizophrenen Formenkreis, schwerer Krankheitsgrad und niedriges Funktionsniveau bei Klinikaufnahme, umfangreiche soziale Probleme, fehlende Arbeitsfähigkeit, wenige soziale Bindungen, Wohnsitzlosigkeit beziehungsweise Aufenthalt in Institutionen [10, 12]. Während der Anteil der Patienten mit einer schizophrenen Psychose an stationären Behandlungen gemäß früherer Untersuchungen in den letzten dreißig Jahren auf $20 \%$ gesunken ist [2], macht deren Anteil an langen und häufigen Hospitalisationen sowohl entsprechend der hier dargestellten Resultate als auch entsprechend kürzlich publizierter Ergebnisse [15] einen unverändert hohen Anteil aus.

Gemessen an der Effektstärke haben sich neben klinischen Faktoren insbesondere die Finanzierungssituation vor Klinikaufnahme, die Wohnsituation nach Entlassung sowie behandlungsbezogene Variablen als besonders relevant herausgestellt. Soziale Faktoren, insbesondere eine prekäre Wohn- und Finanzierungssituation, tragen offenbar wesentlich zur Dauer der Aufenthalte auf Akutstationen bei. Außerdem diskussionswürdig erscheint die Assoziation des Besuchs verschiedener Therapien (zum Beispiel Arbeits- und Ergotherapie) mit der langen Aufenthaltsdauer. Auch wenn dies einerseits für einen therapeutischen Auftrag sprechen könnte, der den Behandlungszeitraum verlängern würde, ist es wahrscheinlicher eine Folge des andauernden 
Aufenthaltes wegen fehlender Entlassungsfähigkeit. Die hohe Relevanz der Variablen „Involvierung der Sozialarbeit“ sowie „Entlassung in eine betreute Wohnform“ spricht für diese Interpretation.

Dies wird durch die Ergebnisse des Regressionsmodells unterstützt, welches unter anderem diese Variablen mit hohen Odds ratios einschließt. Die Interpretierbarkeit dieses Modells ist wegen des Problems der Multikollinearität bei der Vielzahl eingeschlossener Variablen jedoch eingeschränkt. Die Varianzaufklärung für eine lange Aufenthaltsdauer lag bei $10 \%$ für soziodemographische, $15 \%$ für klinische und 19\% für behandlungsbezogene Faktoren. Die gesamte Varianzaufklärung von über 43\% ist ein hohes Ergebnis gegenüber früheren Studien zu Einflussfaktoren auf die Aufenthaltsdauer [8] wobei insbesondere der Anteil aufgeklärter Varianz durch klinische Faktoren (hier insbesondere Schweregrad bei Aufnahme) auffällt.

Ein Drittel der hier untersuchten Patienten mit langer Aufenthaltsdauer wird in eine betreute Wohneinrichtung entlassen, gegenüber weniger als $10 \%$ der Patienten mit einer Aufenthaltsdauer unter einem Monat. Diese Patienten weisen eine nochmals erhöhte Verweildauer auf und unterscheiden sich von den anderen Patienten in einer Reihe von Variablen, die auf eine desintegrierte soziale Situation, eine längere psychiatrische Behandlungsgeschichte, jedoch nicht unmittelbar auf einen höheren Erkrankungsgrad schließen lassen. Schwierigkeiten, eine geeignete Wohnform für die oben beschriebene Patientengruppe zu finden, dürften in der Praxis neben dem schweren Erkrankungsgrad wesentlich zur hohen Inanspruchnahme akutstationärer Behandlungskapazitäten beitragen. Ein mangelndes Angebot an betreuten Wohnformen in quantitativer Hinsicht kann im hier untersuchten Versorgungsgebiet keine Rolle spielen, da im Kanton Zürich im Vergleich zu anderen Regionen ein überproportionales Angebot besteht, welches gut 50\% der Versorgungskosten schizophrener Patienten generiert [19]. Vielmehr scheint das Problem auf der qualitativen Seite zu liegen, in dem Sinne, dass Wohneinrichtungen oftmals zu hohe Zugangsvoraussetzungen haben, ein zu hohes Funktionsniveau erwarten oder andererseits zu wenig flexible Unterstützung beziehungsweise Strukturen bieten. Diese Vermutung basiert jedoch auf Erfahrungen aus der klinischen Praxis und kann mit den dargestellten Daten nicht untermauert werden.

Zu weiteren sozialen beziehungsweise auf die Versorgungssituation bezogenen Faktoren, die zu einer längeren Aufenthaltsdauer beitragen, kann hier ebenfalls keine Aussagen getroffen werden. Aus der Literatur ist jedoch bekannt, dass sowohl klinische als auch soziale Probleme das 
Wiederaufnahmerisiko vulnerabler Patienten erhöhen [20, 21]. Für die Gruppe der Patienten, die stationäre Versorgungsstrukturen primär wegen sozialen Angelegenheiten in Anspruch nehmen, insbesondere dem Suchen einer Wohnform, sind alternative (und kostengünstigere) Angebote notwendig $[15,22]$. In einer qualitativen Untersuchung der subjektiven Perspektive von „Heavy usern“ gaben $65 \%$ der Befragten an, dass zumindest ein Teil der Spitalaufenthalte durch verbesserte Krisenintervention mit ärztlichen, pflegerischen beziehungsweise sozialarbeiterischen Hausbesuchen vermeidbar gewesen wäre [11]. Das Angebot einer Krisenintervention durch ein multiprofessionelles Team im häuslichen Umfeld beziehungsweise im Wohnheim kann dazu beitragen, Krisen frühzeitig abzufangen und Klinikaufenthalte zu reduzieren [23-25]. Diese Befunde stellen eine Herausforderung für die Schnittstelle zwischen Kliniken und nachsorgenden Hilfsangeboten dar. Die subjektive Perspektive der Patienten ist bei einer entsprechenden Planung unbedingt einzubeziehen [26]

Diese Studie untersucht selektiv den jeweils längsten Behandlungsfall aller Patienten auf psychiatrischen Akutstationen in einem Untersuchungszeitraum von fünf Jahren. Hierdurch werden eine Reihe Patienten ausgeschlossen, die zum Beispiel im Untersuchungszeitraum ebenfalls lange Aufenthalte auf Akutstationen hatten, deren längster Aufenthalt jedoch auf einer spezialisierten oder einer offenen Station stattfand. Diese Selektion führt möglicherweise zu einer Überschätzung von Patienten mit schizophrener Psychose und niedrigem Funktionsniveau beziehungsweise einer Unterschätzung komplex traumatisierter Patienten mit Persönlichkeitsstörung, die auch auf Spezialstationen behandelt werden. Andererseits führt diese Selektion wahrscheinlich auch zu einer Unterschätzung des Ausmaßes der Problematik der langen Aufenthaltsdauer im Zusammenhang mit einer Platzierung in betreute Wohneinrichtungen, da gerade Patienten mit Wohnproblematik bei vorhandener oder wiederhergestellter Absprachefähigkeit im Verlauf auf offene Stationen verlegt werden.

Da für die logistische Regressionsanalyse nochmals die Fälle mit fehlenden Werten in einzelnen Variablen ausgeschlossen wurden, beziehen sich die Ergebnisse dieser Analyse auf eine kleinere Stichprobe. Außer der Verteilung der Aufenthaltsdauer auf die drei Kategorien, die fast identisch ist, wurde keine Drop-out-Analyse vorgenommen. Dies könnte zu unterschiedlichen Analysegruppen geführt haben, obwohl das Auswahlkriterium „keine fehlenden Werte“ keinen systematischen Selektionseffekt nahelegt. Zudem bilden sich die Ergebnisse der multivariaten Analyse auch in den bivariaten Ergebnissen unter Einschluss aller Patienten ab. Die Ergebnisse der Regressionsanalyse 
werden durch das oben genannte Problem der Multikollinearität eingeschränkt, welches aufgrund der Vielzahl eingeschlossener Variablen schwer zu kontrollieren ist. Aus diesem Grund wurden die relativen Effektstärken in der bivariaten Auswertung hinzugezogen, um die Relevanz der einzelnen Variablen besser beurteilen zu können.

Die Ergebnisse zeigen, dass ein substanzieller Anteil der Patienten mit langer Aufenthaltsdauer durch eine Reihe von Variablen, die aus der „Heavy user“-Forschung bekannt sind, gut beschrieben werden kann. Der in der patientenbezogenen Auswertung bereits sehr hohe Ressourcenbedarf dürfte in der Praxis erheblich größer sein, da die Patienten mit der längsten Aufenthaltsdauer im hier ausgewerteten Einzelfall auch eine hohe kumulative Aufenthaltsdauer und eine hohe Fallzahl aufweisen.

\section{$\underline{\text { Schlussfolgerungen }}$}

Ein Drittel der Patienten, die mindestens einen Aufenthalt von mehr als 2 Monaten auf psychiatrischen Akutstationen benötigen tritt in betreute Wohneinrichtungen aus. Bei einem substanziellen Teil handelt es sich um Patienten, die auch als „Heavy user" bezeichnet werden. Neben klinischen Aspekten spielen wahrscheinlich soziale Faktoren, insbesondere die Wohnsituation, eine wesentliche Rolle dafür, dass sich diese Patienten lange auf Akutstationen aufhalten. Dies stellt eine Herausforderung für die Versorgung an der Schnittstelle zwischen Kliniken und nachsorgenden Hilfsangeboten dar.

\section{Interessenkonflikte}

Keine angegeben.

\section{Literatur}

${ }^{1}$ Richter D. Qualitätsindikatoren für die psychiatrische Versorgung - Eine Übersicht über Kriterien, Methoden und Probleme. Krankenhauspsychiatrie 2004; 15: 104-113

${ }^{2}$ Lay B, Nordt C, Rössler W. Trends in psychiatric hospitalisation of people with schizophrenia: a register-based investigation over the last three decades. Schizophr Res 2007; 97: 68-78

${ }^{3}$ SpiessI H, Klein HE. Eine untere Grenze der Verweildauer ist erreicht! Psychiatr Prax 2008; 35: 269270

${ }^{4}$ Kuhl HC. Stationäre Psychiatrie in der Schweiz 2000-2006. Obsan Arbeitsdokument 31. Neuchatel: OBSAN; 2008 
${ }^{5}$ Warnke I, Rössler W, Herwig U. Does psychopathology at admission predict the length of inpatient stay in psychiatry? Implications for financing psychiatric services. BMC Psychiatry 2011; 11: 120

${ }^{6}$ Herwig U, Warnke I, Rössler W. Psychopathologische Syndrome gemäss AMDP-System als Grundlage fur Fallgruppierungen in der Psychiatrie. Psychiatr Prax 2009; 36: 320-326

${ }^{7}$ Zhang J, Harvey C, Andrew C. Factors associated with length of stay and the risk of readmission in an acute psychiatric inpatient facility: a retrospective study. Aust N Z J Psychiatry 2011; 45: 578-585

${ }^{8}$ Richter D. Die Dauer der stationären psychiatrischen Behandlung - Eine Übersicht über Methodik, Einflussfaktoren und Auswirkungen. Fortschr Neurol Psychiatr 2001; 69: 19-31

${ }^{9}$ Kent S, Fogarty M, Yellowlees P. A review of studies of heavy users of psychiatric services. Psychiatr Serv 1995; 46: 1247-1253

${ }^{10}$ Spiessl H, Hubner-Liebermann B, Binder H et al. "Heavy Users" in einer psychiatrischen Klinik Eine Kohortenstudie mit 1811 Patienten über fünf Jahre. Psychiatr Prax 2002; 29: 350-354

${ }^{11}$ Roick C, Heider D, Stengler-Wenzke K et al. Analyse starker Inanspruchnahme stationärpsychiatrischer Versorgung aus drei unterschiedlichen Perspektiven. Psychiatr Prax 2004; 31: 241 249

${ }^{12}$ Lay B, Lauber C, Rössler W. Prediction of in-patient use in first-admitted patients with psychosis. Eur Psychiatry 2006; 21: 401-409

${ }^{13}$ Lucas B, Harrison-Read P, Tyrer P et al. Costs and characteristics of heavy inpatient service users in outer London. Int J Soc Psychiatry 2001; 47: 63-74

${ }^{14}$ Roick C, Gartner A, Heider D et al. Heavy user psychiatrischer Versorgungsdienste - Ein Überblick über den Stand der Forschung. Psychiatr Prax 2002; 29: 334-342

${ }^{15}$ Stulz N, Bielinski D, Junghan UM et al. Stationärer Heavy Use und die Inanspruchnahme ambulanter Dienste in einem Schweizerischen Versorgungssystem. Psychiatr Prax 2012; 39: 332338

${ }^{16}$ Kyle T, Dunn JR. Effects of housing circumstances on health, quality of life and healthcare use for people with severe mental illness: a review. Health Soc Care Community 2008; 16: 1-15

${ }^{17}$ Guy W. Assessment Manual for Psychopharmacology - Revised. Rockville: U.S. Department of Health Education and Welfare; 1976

${ }^{18}$ APA. Diagnostic and Statistical Manual of Mental Disorders, 4th Edition. Washington, DC: American Psychiatric Association; 1994 
${ }^{19}$ Salize HJ, McCabe R, Bullenkamp J et al. Cost of treatment of schizophrenia in six European countries. Schizophr Res 2009; 111: 70-77

Formatiert: Deutsch (Schweiz)

${ }^{20}$ Warnke I, Nordt C, Ajdacic-Gross $\vee$ et al. Klinische und soziale Risikofaktoren fur Wiederaufnahmen in die stationäre Psychiatrie bei Patienten mit Schizophrenie: Eine Langzeitanalyse. Neuropsychiatr

2010; 24: 243-251

${ }^{21}$ Durbin J, Lin E, Layne $\mathrm{C}$ et al. Is readmission a valid indicator of the quality of inpatient psychiatric care? J Behav Health Serv Res 2007; 34: 137-150

${ }^{22}$ Roick C, Heider D, Kilian R et al. Patienten mit starker Inanspruchnahme stationär-psychiatrischer Versorgung - Eine Analyse von Krankenkassendaten aus der Stadt Leipzig. Psychiatr Prax 2002; 29: $343-349$

${ }^{23}$ Barker V, Taylor M, Kader I et al. Impact of crisis resolution and home treatment services on user experience and admission to psychiatric hospital. The Psychiatrist 2011; 35: 106-110

${ }^{24}$ Mueser KT, Bond GR, Drake RE et al. Models of community care for severe mental illness: a review of research on case management. Schizophr Bull 1998; 24: 37-74

${ }^{25}$ Marshall M, Lockwood A. Assertive community treatment for people with severe mental disorders. Cochrane Database Syst Rev 1998, 2; CD001089

${ }^{26}$ Steffen S, Kosters M, Becker T et al. Discharge planning in mental health care: a systematic review of the recent literature. Acta Psychiatr Scand 2009; 120: 1-9

\section{Anhang: Tabellen und Abbildungslegenden}

Tabelle 1: Gruppenvergleich kategoriale Variablen (Angaben in \%)

\begin{tabular}{|c|c|c|c|c|c|c|c|c|}
\hline \multirow{2}{*}{ Variable } & \multirow{2}{*}{$\begin{array}{l}\text { Gesamt- } \\
\text { stichprobe }\end{array}$} & \multicolumn{3}{|c|}{ Aufenthaltsdauer in Tagen } & \multirow{2}{*}{$\mathrm{Chi}^{2}$} & \multirow{2}{*}{ df } & \multirow{2}{*}{ p-Wert } & \multirow{2}{*}{ Cramer $\mathrm{V}^{*}$} \\
\hline & & $<31$ & $31-60$ & $>60$ & & & & \\
\hline $\mathrm{N}(\%)$ & $3928(100)$ & $2514(64)$ & $758(19)$ & $656(17)$ & & & & \\
\hline Frauen & 42,4 & 42,0 & 41,6 & 44,8 & 1,951 & 2 & 0,377 & 0,023 \\
\hline Schweizer & 62,8 & 60,3 & 65,5 & $68,9^{*}$ & 28,148 & 4 & $<0,0001$ & 0,080 \\
\hline Ledig & 54,3 & 52,2 & 52,8 & $63,5^{*}$ & 30,018 & 4 & $<0,0001$ & 0,099 \\
\hline \multicolumn{9}{|l|}{ Erwerbstätigkeit } \\
\hline voll vor Aufnahme & 17,6 & $22,0^{*}$ & $11,0^{*}$ & $8,6^{*}$ & \multirow{2}{*}{128,594} & \multirow{2}{*}{6} & \multirow{2}{*}{$<0,0001$} & \multirow{2}{*}{0,179} \\
\hline nicht vor Aufnahme & 56,5 & $51,5^{*}$ & 61,6 & $69,2^{*}$ & & & & \\
\hline voll nach Entlassung & 13,6 & $18,1^{*}$ & $7,3^{*}$ & $3,7^{*}$ & \multirow{2}{*}{142,218} & \multirow{2}{*}{6} & \multirow{2}{*}{$<0,0001$} & \multirow{2}{*}{0,185} \\
\hline nicht nach Entlassung & 61,0 & $56,1^{*}$ & 66,1 & $73,3^{*}$ & & & & \\
\hline
\end{tabular}




\begin{tabular}{|c|c|c|c|c|c|c|c|c|}
\hline Lebensunterhalt & & & & & & & & \\
\hline eigener Verdienst & 29,1 & $36,2^{*}$ & $19,8^{*}$ & $15,0^{*}$ & \multirow{2}{*}{207,319} & \multirow{2}{*}{6} & \multirow{2}{*}{$<0,0001$} & \multirow{2}{*}{0,261} \\
\hline Invalidenrente & 40,0 & $30,8^{*}$ & $50,1^{*}$ & $60,0^{*}$ & & & & \\
\hline Hauptdiagnosen & & & & & \multirow{5}{*}{303,269} & \multirow{5}{*}{12} & \multirow{5}{*}{$<0,0001$} & \multirow{5}{*}{0,274} \\
\hline F1 & 22,6 & $26,6^{*}$ & $19,0^{*}$ & $11,3^{*}$ & & & & \\
\hline $\mathrm{F} 2$ & 33,2 & $25,3^{*}$ & $41,8^{*}$ & $53,8^{*}$ & & & & \\
\hline F3 & 17,4 & 16,9 & 20,4 & 15,9 & & & & \\
\hline $\mathrm{F} 4$ & 14,3 & $18,4^{*}$ & $7,4^{*}$ & $6,3^{*}$ & & & & \\
\hline Voraufenthalte & & & & & \multirow{4}{*}{371,988} & \multirow{4}{*}{4} & \multirow{4}{*}{$<0,0001$} & \multirow{4}{*}{0,322} \\
\hline Erstaufenthalt & 54,1 & $64,7^{*}$ & $41,6^{*}$ & $28,8^{*}$ & & & & \\
\hline 1-4 Aufenthalte & 31,1 & $25,9^{*}$ & $39,7^{*}$ & $40,6^{*}$ & & & & \\
\hline 5 und mehr & 14,8 & $9,4^{*}$ & $18,7^{*}$ & $30,6^{*}$ & & & & \\
\hline \multicolumn{9}{|l|}{ Aufnahme } \\
\hline mit FFE & 53,2 & $58,2^{*}$ & $45,9^{*}$ & $42,4^{*}$ & 71,524 & 2 & $<0,0001$ & 0,128 \\
\hline aus Institution & 19,9 & $18,0^{*}$ & 22,6 & $23,7^{*}$ & \multirow{2}{*}{32,737} & \multirow{2}{*}{6} & \multirow{2}{*}{$<0,0001$} & \multirow{2}{*}{0,088} \\
\hline wohnsitzlos & 4,4 & $3,5^{*}$ & 5,6 & $6,3^{*}$ & & & & \\
\hline $\begin{array}{l}\text { Zuweisung durch } \\
\text { Psychiater }\end{array}$ & 35,2 & 34,0 & 36,4 & 38,3 & \multirow{3}{*}{43,541} & \multirow{3}{*}{10} & \multirow{3}{*}{$<0,0001$} & \multirow{3}{*}{0,109} \\
\hline $\begin{array}{l}\text { aus nicht- } \\
\text { psychiatrischem } \\
\text { Krankenhaus }\end{array}$ & 31,1 & $34,2^{*}$ & 28,5 & $22,3^{*}$ & & & & \\
\hline aus $\mathrm{PIA} / \mathrm{TK}^{\circ}$ & 3,1 & 2,8 & 2,3 & $4,9^{*}$ & & & & \\
\hline \multicolumn{9}{|l|}{ Aufenthalt } \\
\hline Verlegung & 4,5 & $2,8^{*}$ & 5,7 & $9,8^{*}$ & 60,612 & 2 & $<0,0001$ & 0,139 \\
\hline Zwangsmaßnahme ${ }^{\circ \circ}$ & 12,1 & $9,3^{*}$ & 11,7 & $23,0^{*}$ & 87,438 & 2 & $<0,0001$ & 0,169 \\
\hline Pharmakotherapie & 88,5 & $83,7^{*}$ & $95,5^{*}$ & $97,1^{*}$ & 128,778 & 2 & $<0,0001$ & 0,163 \\
\hline Arbeitstherapie & 21,6 & $8,3^{*}$ & $40,2^{*}$ & $44,3^{*}$ & 389,876 & 2 & $<0,0001$ & 0,409 \\
\hline Ergo-/Musiktherapie & 41,6 & $22,7^{*}$ & $66,0^{*}$ & $69,8^{*}$ & 530,216 & 2 & $<0,0001$ & 0,431 \\
\hline $\begin{array}{l}\text { Physiotherapie/Bewegun } \\
\text { g }\end{array}$ & 33,8 & $18,4^{*}$ & $54,1^{*}$ & $59,6^{*}$ & 390,847 & 2 & $<0,0001$ & 0,389 \\
\hline Sozialarbeit & 28,3 & $17,3^{*}$ & $40,8^{*}$ & $48,5^{\star}$ & 233,086 & 2 & $<0,0001$ & 0,310 \\
\hline Begutachtung & 1,5 & 0,8 & 1,9 & $3,8^{*}$ & 16,938 & 2 & $<0,0001$ & 0,099 \\
\hline Wichtigste Behandlung & & & & & \multirow{4}{*}{229,846} & \multirow{4}{*}{6} & \multirow{4}{*}{$<0,0001$} & \multirow{4}{*}{0,235} \\
\hline Krisenintervention & 19,3 & $24,5^{*}$ & $12,9^{*}$ & $7,2^{*}$ & & & & \\
\hline Psychotherapie & 3,1 & $2,1^{*}$ & $5,4^{*}$ & 3,8 & & & & \\
\hline Pharmakotherapie & 13,3 & $8,9^{*}$ & $18,9^{*}$ & $23,8^{*}$ & & & & \\
\hline \multicolumn{9}{|l|}{ Entlassung } \\
\hline $\begin{array}{l}\text { Entscheid } \\
\text { einvernehmlich }\end{array}$ & 76,0 & $70,5^{\star}$ & 85,9 & $86,1^{*}$ & 118,935 & 4 & $<0,0001$ & 0,146 \\
\hline
\end{tabular}




\begin{tabular}{|c|c|c|c|c|c|c|c|c|}
\hline ins betreute Wohnen & 15,1 & $8,3^{*}$ & $20,2^{*}$ & $31,7^{*}$ & \multirow{2}{*}{289,338} & \multirow{2}{*}{6} & \multirow{2}{*}{$<0,0001$} & \multirow{2}{*}{0,310} \\
\hline andere Klinik & 10,6 & $12,2^{*}$ & $7,7^{*}$ & $7,8^{*}$ & & & & \\
\hline \multicolumn{5}{|l|}{ Behandlung durch } & \multirow{4}{*}{177,556} & \multirow{4}{*}{10} & \multirow{4}{*}{$<0,0001$} & \multirow{4}{*}{0,216} \\
\hline Psychiater/Therapeut & 38,4 & $35,0^{*}$ & $45,4^{*}$ & 43,1 & & & & \\
\hline anderer Arzt & 15,0 & $17,6^{*}$ & $10,6^{*}$ & $10,4^{*}$ & & & & \\
\hline $\mathrm{PIA} / \mathrm{TK}^{\circ}$ & 14,7 & $11,2^{*}$ & 16,6 & $25,5^{\star}$ & & & & \\
\hline \multicolumn{5}{|l|}{ Versicherungsklasse } & \multirow{4}{*}{6,101} & \multirow{4}{*}{4} & \multirow{4}{*}{0,192} & \multirow{4}{*}{0,042} \\
\hline Allgemein & 100 & 63,8 & 19,2 & 17,0 & & & & \\
\hline Halbprivat & 100 & 68,8 & 23,4 & 7,8 & & & & \\
\hline Privat & 100 & 70,2 & 19,1 & 10,6 & & & & \\
\hline
\end{tabular}

\# als Maß für die Effektstärke: Gruppe $<31$ gegenüber $>60$

* standardisierte Residuen $>2$

- Institutsambulanz oder Tagesklinik der Psychiatrischen Universitätsklinik Zürich

$\circ$ Isolation, Fixierung und/oder Zwangsmedikation

Tabelle 2: Assoziierte Faktoren mit der Aufenthaltsdauer: binäre logistische Regressionsanalyse

\begin{tabular}{|c|c|c|c|c|}
\hline $\begin{array}{l}\text { Block/ } \\
\text { Variablen }\end{array}$ & $\operatorname{Exp}(B)$ & $\operatorname{Exp}(95 \% \mathrm{Cl})$ & $\begin{array}{l}\text { Nagelkerke } R^{2} \\
\text { (Differenz) }\end{array}$ & $\mathbf{p}$ \\
\hline Konstante & 0,010 & & & $<0,0001$ \\
\hline \multicolumn{5}{|l|}{$\begin{array}{l}\text { Soziodemographische } \\
\text { Faktoren* }\end{array}$} \\
\hline IV-/AHV-Rente & 1,793 & $1,261-2,549$ & $0,091(0,09)$ & 0,001 \\
\hline Ledig & 1,449 & $1,034-2,031$ & $0,103(0,01)$ & 0,031 \\
\hline \multicolumn{5}{|l|}{ Klinische Faktoren } \\
\hline Schweregrad bei Aufnahme & 1,777 & $1,403-2,250$ & $0,219(0,11)$ & $<0,0001$ \\
\hline $\begin{array}{l}\text { Hauptdiagnose } \\
\text { Schizophrenie }\end{array}$ & 1,705 & $1,212-2,398$ & $0,251(0,03)$ & 0,002 \\
\hline $\begin{array}{l}\text { Funktionsniveau bei } \\
\text { Aufnahme }\end{array}$ & 0,987 & $0,967-0,990$ & $0,259(0,01)$ & $<0,0001$ \\
\hline \multicolumn{5}{|l|}{$\begin{array}{l}\text { Behandlungsbezogene } \\
\text { Faktoren }\end{array}$} \\
\hline Mehr als 5 Aufnahmen & 3,369 & $2,189-5,186$ & $0,304(0,05)$ & $<0,0001$ \\
\hline Sozialarbeit involviert & 3,764 & $2,474-5,726$ & $0,351(0,05)$ & $<0,0001$ \\
\hline
\end{tabular}




\begin{tabular}{|l|l|l|l|l|}
\hline Aufnahme per FFE & 0,430 & $0,303-0,610$ & $0,374(0,02)$ & $<0,0001$ \\
\hline $\begin{array}{l}\text { Verlegung während } \\
\text { Aufenthalt }\end{array}$ & 3,356 & $1,817-6,195$ & $0,387(0,02)$ & $<0,0001$ \\
\hline Zwangsmaßnahme & 1,906 & $1,190-3,052$ & $0,393(0,01)$ & 0,007 \\
\hline $\begin{array}{l}\text { Entlassungsbezogene } \\
\text { Faktoren }\end{array}$ & & & & \\
\hline Entlassung ins Wohnheim & 2,723 & $1,787-4,150$ & $0,413(0,02)$ & $<0,0001$ \\
\hline Behandlung durch PUK & 2,784 & $1,760-4,404$ & $0,423(0,01)$ & $<0,0001$ \\
\hline $\begin{array}{l}\text { Behandlung durch } \\
\text { niedergelassenen } \\
\text { Psychiater }\end{array}$ & 1,869 & $1,289-2,710$ & $0,432(0,01)$ & 0,001 \\
\hline
\end{tabular}

Anmerkungen: Gesamtzahl mit vollständigem Datensatz: $\mathrm{N}=1.761$, Aufenthaltsdauer $>60$ Tage: $\mathrm{N}=271$ (15\%); $<31$ Tage: $\mathrm{N}=1.158(66 \%)$.

Modellstatistik: Chi-Quadrat $=446.335, \mathrm{df}=13, \mathrm{p}<0,0001$

${ }^{*}$ Die Variablen Schweizer und Alter erreichten am Ende der Prozedur kein signifikantes Niveau mehr, sodass sie wieder aus dem Modell entfernt wurden.

Tabelle 3: Assoziationen mit der Variablen „Entlassung ins Wohnheim“ (ab Signifikanzniveau p<0,01; Angaben in \%)

\begin{tabular}{|c|c|c|c|c|c|}
\hline Variable & $\begin{array}{l}\text { Wohnheim } \\
(\mathrm{N}=208)\end{array}$ & $\begin{array}{l}\text { Anderes } \\
(\mathrm{N}=448)\end{array}$ & $\mathrm{Chi}^{2}$ & df & $p$ \\
\hline verheiratet & $7,9^{*}$ & 16,6 & 13,920 & 2 & 0,001 \\
\hline aus Institution & $46,5^{*}$ & $12,9^{*}$ & 87,752 & 3 & $<0,0001$ \\
\hline nicht erwerbstätig & $81,5^{*}$ & 63,4 & 31,681 & 3 & $<0,0001$ \\
\hline eigener Verdienst & $3,4^{*}$ & $20,8^{*}$ & 30,282 & 3 & $<0,0001$ \\
\hline mind. 5 Voraufenthalte & $40,6^{*}$ & 26,0 & 15,711 & 2 & $<0,0001$ \\
\hline Hauptdiagnose F1 & $15,9^{*}$ & 9,2 & \multirow{3}{*}{43,959} & \multirow{3}{*}{6} & \multirow{3}{*}{$<0,0001$} \\
\hline Hauptdiagnose F2 & 58,2 & 51,8 & & & \\
\hline Hauptdiagnose F3 & $5,3^{*}$ & $20,8^{*}$ & & & \\
\hline $\begin{array}{l}\text { Entlassungsentscheid durch } \\
\text { Patient (nicht einvernehmlich) }\end{array}$ & $1,0^{*}$ & $9,0^{*}$ & 18,423 & 2 & $<0,0001$ \\
\hline Sozialdienst & 56,7 & 44,4 & 6,616 & 1 & 0,007 \\
\hline
\end{tabular}


Abbildung 1: Stetige klinische Variablen: Schweregrad der Erkrankung, Funktionsniveau

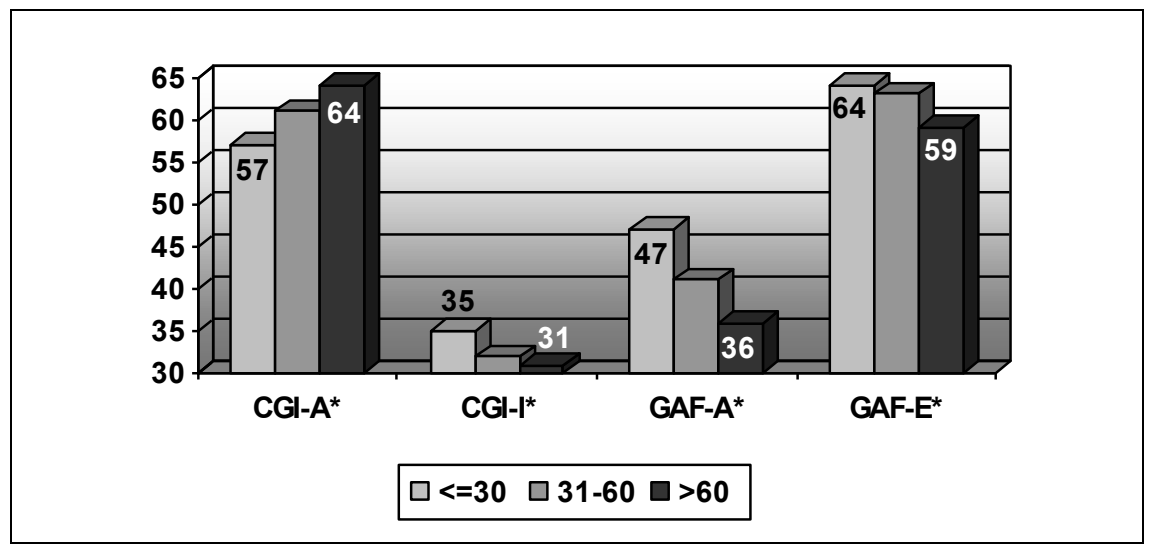

*ANOVA, $p<0,0001$ (Gruppe $>60$ Tage gegenüber Vergleichsgruppen)

CGI-A: Klinischer Schweregrad bei Aufnahme ( $2=$ nicht krank, $5=$ mäßig krank, $8=$ extrem schwer krank; Wert x 10)

CGI--: Zustand bei Entlassung im Vergleich zum Aufnahme ( $2=$ sehr viel besser, $4=$ nur wenig besser, $8=$ sehr viel schlechter; Wert $x 10)$ GAF: Puscher 